\title{
SEARCH FOR EFFECTIVE SYNTHETIC AGENTS AGAINST INFLUENZA VIRUS
}

\author{
SHIGESHI TOYOSHIMA, SEIZABURO KANO, and TAKEO UEDA* \\ Pharmaceutical Institute, Keio University, Tokyo, Japan
}

(Received for publication September 11, 1963)

\begin{abstract}
At latest, all of type-strains of influenza virus have been found to produce only very weak immunity in human body. This finding suggests that the vaccination is rather disadvantageous for the treatment of influenzal diseases and accordingly chemoprophylactic and chemotherapeutic agents against influenza are desired to be created. To date, many researchers have claimed their antiinfluenzal agents by a variety of screening methods. Almost all of these agents, however, have been denied as to their antiinfluenzal activity in animal experiments. Therefore, problems still remain to find antiinfluenzal agents which should be effective in animal experiments, as the precursor of an antiinfluenzal drug.

The authors screened many compounds of various types synthesized by our group as to their antiinfluenzal effect in chorioallantoic membrane culture and in mice. As the results obtained, we found an effective agent on influenza virus in mice, for the first time.

This paper describes the results of the screening tests with many compounds synthesized by our group.
\end{abstract}

\section{METHOD OF SCREENING FOR INFLUENZA VIRUS}

At first, it should be emphasized that the present method should be suitable for the finding of active agents aiming ultimately at antiinfluenzal drugs. There have been so far submitted many screening methods for finding antiinfluenzal agents. These methods fall into the five as follows:

(1) Test by agglutination of blood cells with allantoic fluid of chick embryos infected with influenza virus,

(2) Test employing deembryonated eggs,

(3) Test by tissue culture, employing monkey kidney cells,

(4) Test by chorioallantoic membrane culture of embryonated eggs,

(5) in vivo Test employing mice.

Of these tests, test (2) and (3) may be kept out because of the complexity

* Professor of Pharmaceutical Institute. 
of experimental procedures for routine assay and the difficulty of the procurement of experimental materials. Test (1) may be also negligible because of its too low sensitivity to discern effects of agents. Test (4) and (5) may be taken into consideration to employ for the purpose of finding antiinfluenzal agents. In practice, these two methods have been hithertofore adopted by many researchers for the screening of antiinfluenzal agents.

Criterions may be raised regarding the effect of agents in choriollantoic membrane culture. Regarding this problem, it may be inferred that this effect reveals from the inhibitory action of agents on the virus inside and/or outside membrane cells, and/or the inhibitory effect on the adsorption of the virus onto membrane cells. It is, therefore, difficult to correlate the action site and the action phase of agents from the chorioallantoic membrane culture method. It may be, however, said that this method is useful to judge the effect of test-compounds as the preliminary test for searching antiviral agents, though exceptionally unsuitable for agents which exert their effect after the alteration of their structures in living body. In other words, any compound positive to this test may warrent the secondary test by mice experiments.

On conducting the screening test by chorioallantoic membrane culture, it is important to discern reasonably the effect of test-compounds. As for the discernment, the reduction of HA-titers of the treated group is commonly employed, compared with those of the control.

Tamm(1) considered 75 percent inhibition of agglutination of culture fluid with benzimidazole derivatives as the standard of the screening using chorioallantoic membrane culture. Since the test by the agglutination of blood cells is commonly conducted with $2^{\mathbf{n}}$-dilution, the experimental errors reach $\pm \mathbf{5 0}$ percent. It may be, therefore, said that the value of 75 percent inhibition established by Tamm exceeds the experimental error only to very slight extent. In contrast with the finding of Tamm, we(2) found that some compounds of 2-alkylphenoxyalkanoic acid reduced the agglutination to $1 / 128$ dilution, compared with that of the control. This value of inhibition denotes the effectiveness of agents far more elearly than that of Tamm, and covers the uncertainty of the dicision about the effectiveness depending on experimental errors resulted from the deviation of HA-titers of the virus.

There has not been found any agent which has been significantly confirmed as to its antiinfluenzal effect in mice experiments, to date. Accordingly, it is very difficult to screen compounds of one certain series as to their antiinfluenzal effect by some tentative method, select the most effective one, and employ this compound as the standard, arrange a standard method of screening. We cannot, 
therefore, help arranging a tentative method which is considered reasonable from the view of virology and experimental chemotherapy and revising the method when an effective agent is found.

On the other hand, it is well-known that mice infected with influenza virus show consolidation inside their lungs and die, while some of them survive without any symptom of consolidation ${ }^{(3)}$. It is also recognized that the multiplication of influenza virus in lungs is not parallel with the consolidaton, that is, the virus multiplication takes place without any consolidation and lung consolidation is not always accompanied with the virus multiplication(4). This fact seems to mix up the situation to arrange the method of screening. It may be, therefore, said that both of the survival ratio and the inhibition of consolidation should be taken simultaneously into consideration to decide the effect of screened compounds in mice experiments.

Taken the above virological factors into consideration, we arrange the method of screening for antiinfluenzal agents as follows:

(1) Method of Screening by Chorioallantoic Membrane Culture.

Egg adapted influenza A virus (PR-8 and/or Tokyo-57 strain) is employed for the experiment. Chorioallantoic membrane of 11-15 days embryonated egg is cut of into pieces of $1.0 \mathrm{~cm}$ in diameter. $0.1 \mathrm{cc}$ of $10^{-2}$ dilution of the virus is placed in a test-tube containing $0.8 \mathrm{cc}$ of Hanks solution and a piece of the cut membrane. Immediately after the viral inoculation, $0.1 \mathrm{cc}$ of a sterillized solution of a test-compound is added to the above test-tube. After shaking the culture at $37^{\circ}$ for 18 hours, the viral content of the fluid in the test-tube is estimated by the chickencell agglutination, according to the method of Ledinko(2).

When the agglutination titer of the treated with the compound is below $1 / 100$, the compound is recognized as the effective.

(2) Method of Screening by Mice Experiment.

An amount of $10 \times \mathrm{LD}_{50}$ of the virus is inoculated intranasally into groups of mice, and 30 minutes later, doses corresponding to $1 / 10 \times \mathrm{LD}_{50}$ of the testcompound are injected intraperitoneally into the mice, once to three times a day. After two weeks, survival ratios and consolidation scores of the survived mice are recorded, compared with the control. In the meantime, the amounts of the virus present in the mice are measured by the agglutination test. When a significant difference between the tested group and the control is observed in the survival ratio, the consolidation score and the agglutination, the compound is recognized as the effective.

EXPERIMENTAL RESULTS

Today, it is very difficult to investigate the correlation between effects of an 
antiinfluenzal agent in chorioallantic membrane culture, mice experiment and clinical experiment, because any effective agent on influenza virus has not been found in mice and clinical experiments, to date.

This correlation is considered very important to find antiinfluenzal agents on the way to an antiinfluenzal drug.

Some compounds, for instance, Caprochlorone(5), $\mathrm{ABOB}^{(6)}$ and Xenalamine(7) have been so far claimed as antiviral agents in mice and clinical experiments. The finding with Caprochlorone suggests that the effect in chorioallantoic membrane culture might be associated with that in clinical trials, though both of the effects were very slight. On the other hand, the findings with $A B O B$ and Xenalamine seem to suggest that the effects in mice experiments might reflect on that in clinical trials. According to the reexamination of these agents by our group, however, they were found almost ineffective in both of the chorioallantoic membrane culture and the mice experiments. This result well coincides with that of Jackson and his co-workers ${ }^{(8)}$. It may be, therefore, said that even the relationship between effects of an antiinfluenzal agent in chorioallantoic membrane culture and mice experiment is still in the dark. In order to find an antiinfluenzal agent and clarify the relationship between its effects in chorioallantoic membrane culture and mice experiment, we conducted the following experiments.

Our group has synthesized a lot of compounds to search antiviral agents these fourteen years. Of these compounds, about one thousand compounds have been screened as to their effect on influenza $A$ virus in chorioallantoic culture.

For this experiment, four solutions of $2 \times 10^{-4}, 10^{-4}, 2 \times 10^{-5}$ and $10^{-5}$ mole for each of the compounds, were prepared and used as the test solutions. The other experimental procedures were the same to those in described in the above.

In the Table, $A$ denotes the effectiveness showing $x=1$ in $2 \times 10^{-4} \sim 10^{-4}$ molar solution and $x=1 \sim 3$ in $2 \times 10^{-5} \sim 10^{-5}$ molar solution, while $B$, the effectiveness showing $x=1 \sim 2$ in $2 \times 10^{-4} \sim 10^{-4}$ molar solution and $x>3$ in $2 \times 10^{-5} \sim 10^{-5}$ molar solution. All of the compounds having the effect weaker than $B$ are omitted in the Table.

As can be seen from the Table, 1-( $p$-decylphenyl)-3-dimethylamino-1-propanol hydrochloride and 6-propyl-3-mercapto-5-hydroxy-as-triazine were found to have A-effect, while all of the other compounds listed, to have B-effect.

of the reference compounds, Caprochlorone was observed to have only a very weak effect and both of $A B O B$ and Xenalamine not to have any effect. At next, all of the compounds listed in Table 1, were screened as to their in vivo effect 


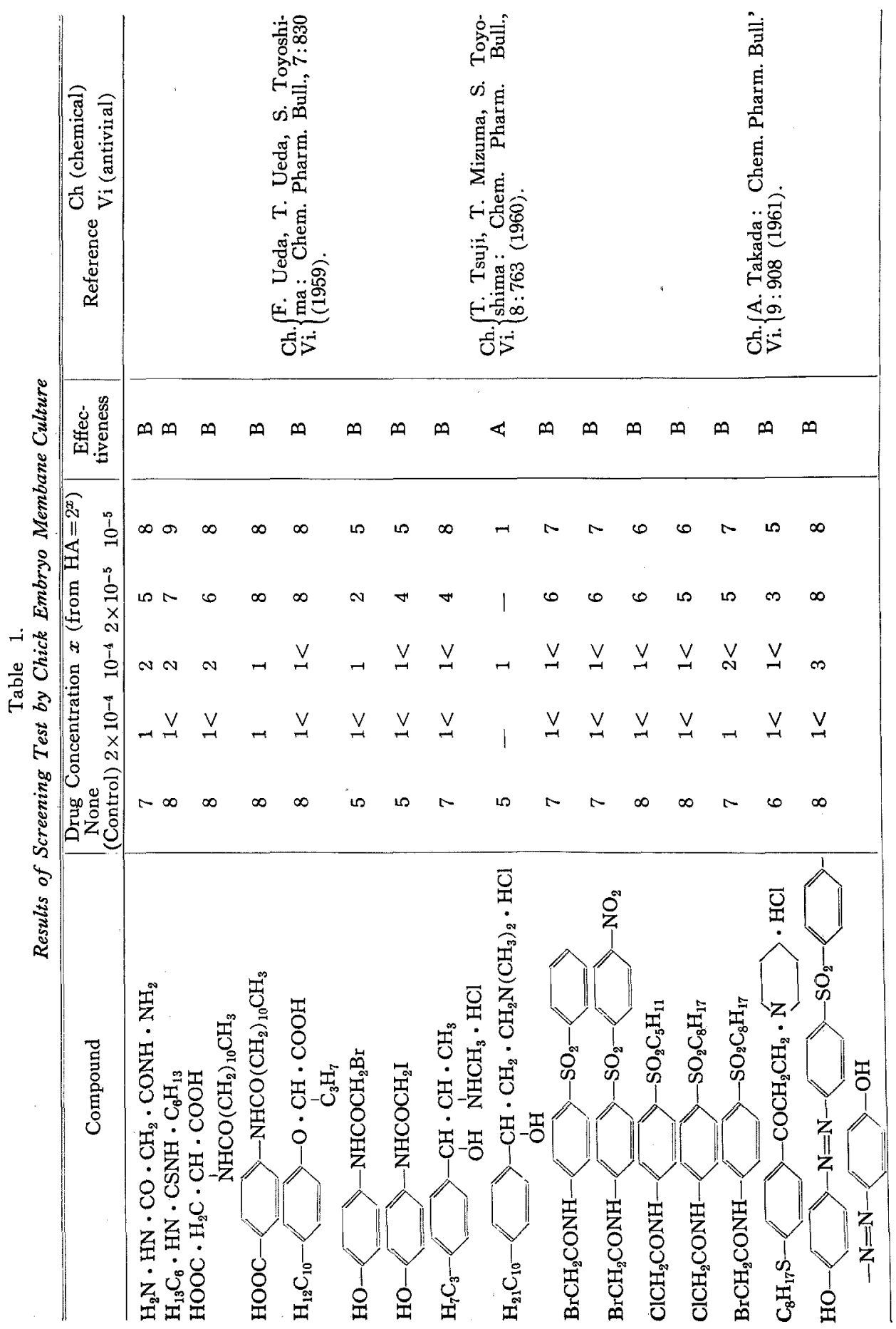




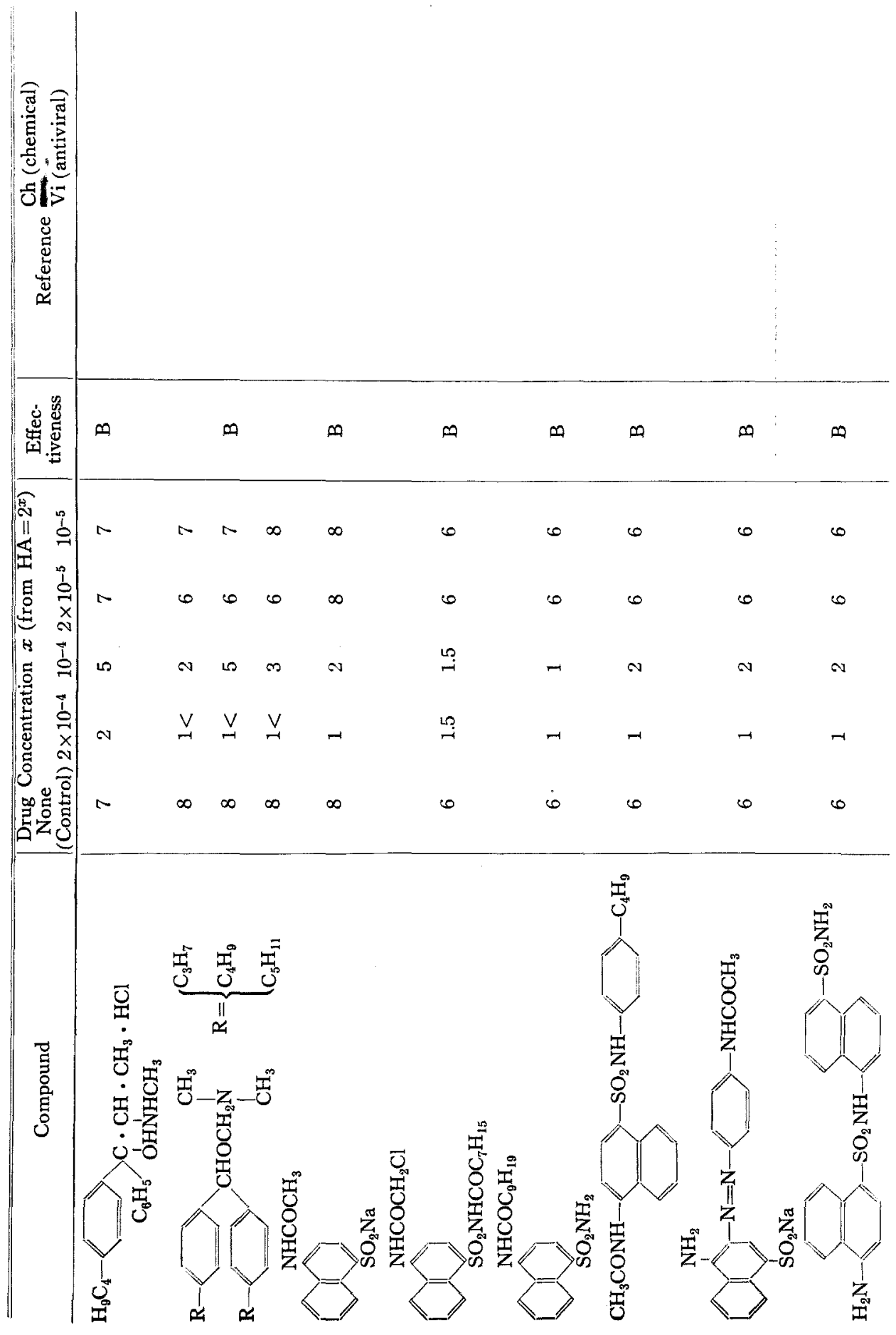




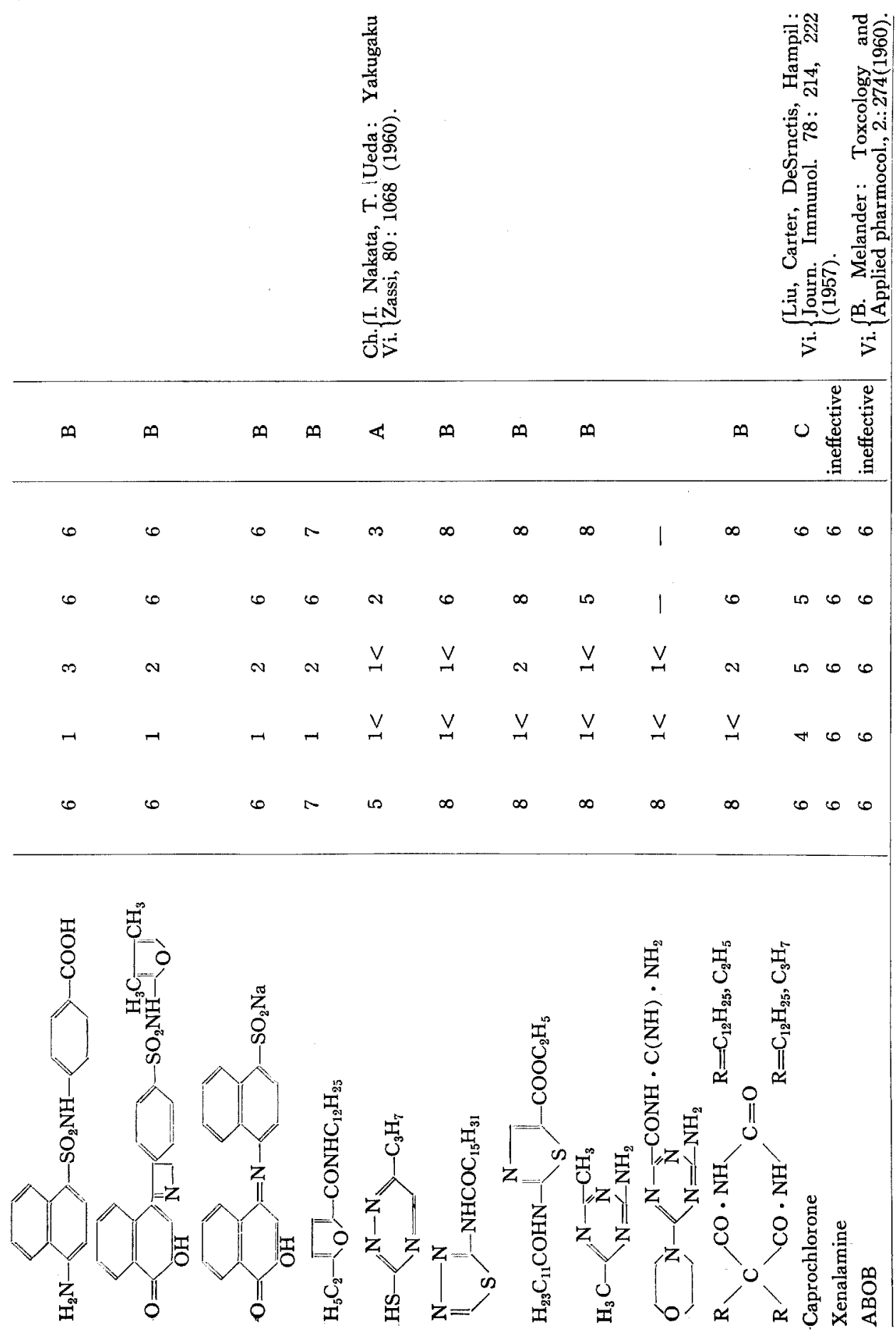


on PR-8 strain and/or Tokyo-57/ Adachi/2 strain in mice. For the experiments, the D.D. strain mice of 9-11 gram in body weight were employed. The other experimental procedures were the same to those described in the above.

As the results obtained, 4-amino-6-morpholino-s-triazine-2-carboxyguanidide was found to show a significant effect on the virus, while all of the other compounds did not exert any significant effect.

The former compound was further surveyed as to its effect as follows. As the viral material, Tokyo-57/ Adachi/2 strain of influenza A virus was used, after it had been adapted to mice with serial passages. For the experiment the D.D. strain mice of 9-11 gram in body weight were employed. Virus dilutions in concentration of $10^{-1} \sim 10^{-3} \quad\left(L_{50}=10^{-3}\right)$ were inoculated intranasally into mice and 30 minutes later, each mouse was injected with a single dose of the compound corresponding to $1 / 3 \times \mathrm{LD}_{50}(=500 \mathrm{mg} / \mathrm{kg})$.

After two weeks, the survival ratio and the consolidation score were caluculated by comparing the treated group with control group. The experimental results are shown in Table 2.

Table 2.

\begin{tabular}{c|cc}
\hline \hline Virus dilution* & Treated group & Effect \\
\hline $10^{-1}$ & $5 / 5 * *(4.4 * * *)$ & $5 / 5(4.75)$ \\
$10^{-2}$ & $10 / 12(3.5)$ & $10 / 10(3.5)$ \\
$10^{-3}$ & $6 / 27(2.0)$ & $13 / 30(3.0)$ \\
\hline
\end{tabular}

* $\mathrm{LD}_{50}$ of the virus is $10^{-3}$ dilution.

** Mice died, total mice used.

*** Consolidation Score.

As can be seen from the Table, it may be said that the compound was effective on the virus. On the other hand, Caprochlorone, $\mathrm{ABOB}$ and Xenalamine hydrochloride selected as the reference compounds did not show any significant effect on the virus by our repeated tests. It may be, therefore, claimed that our compound is the beginning which has been confirmed as to its significant antiinfluenzal in vivo effect in mice.

\section{DISCUSSION}

As described in the experimental part, 4-amino-6-morpholino-s-triazine-2carboxyguanidide was found to exert a significant effect in influenza $A$ virus in mice. Even if the compound is effective on the inoculation of the virus amount corresponding to $\mathrm{LD}_{50}$, this effect might reveal the possibility of chemotherapy on influenza at one certain phase of the viral multiplication, because the natural 
infection of influenza virus in human body takes place in a far lower concentration than the $\mathrm{LD}_{50}$ of the virus titrated.

On the other hand, we ${ }^{(9)}$ found 4-acetaminonaphthalene-1-lauroylsulfonamide (PANS-610) as a chemotherapeutic drug against Japanese B encephalitis, which has been used throughout Japan, since 1956. Our experience with this drug is instructive for the clinical evaluation of antiviral agents. According to our findings as for PANS-610, this drug having a significant effect on the Nakayama strain of Japanese $B$ encephalitis virus in mice, exerted a clinical effect to a certain extent, while PANS-25(10) and PANS-326(10) having slight in vivo effect on the boarderline of plus and minus, did not show any clear-cut clinical effect. This finding suggests that any agent showing at least a significant in vivo effect in mice might be promising for clinical trial. Our finding with 4-amino-6morpholino-s-triazine-2-carboxyguanidide encourages us to advance the antiinfluenzal research program.

At next, of the compounds synthesized by our group the thirty-three compounds were found effective on the virus in chorioallantoic membrane culture, according to our screening method which was more discernable than that of Tamm. However, only 4-amino-6-morpholino-s-triazine-2-carboxyguanidide was found significantly effective in mice experiments, while any of the other compounds was ineffective. This is the first case, in which the compound having a remarkable effect on influenza virus in chorioallantoic membrane culture showed also a in vivo effect in mice.

This finding appearently shows that the effect in chorioallantoic membrane culture might be parallel with that in mice. It is, however, natural that the availability of the chorioallantoic membrane method should be restricted to a narrow range for the purpose of the screening of chemotherapeutic drugs, since this method has been originally devised to investigate the viral multiplication process. This method is considered unavailable for the finding of any agent which exerts an antiviral effect after the alteration of it structure in living body, and impossible to cover the distribution of agents and the interference of components in living body to agents. On the contrary, the mice experiment is useful as the approach to the clinical experiment, since it is concerned with the infection with virus and the chemotherapy with agents in mice.

It may be, therefore, said that antiinfluenzal agents had better be, at first, directly screened by mice experiments, then by monkey experiments and finally by human experiments.

We reexamined the effect of the agents which have been so far claimed to have antiinfluenzal effect in eggs, mice and human experimęnts. 
Liu et al.(5) reported that Caprochlorone showed an effect in the deembryonated eggs, mice and clinical experiments. This agent, however, was later abandoned because of the side-action of such dermatitis as rash. According to our reexamination with this agent, it is found very slightly effective on PR-8 strain in chorioallantoic membrane culture and almost ineffective in mice. We cannot evaluate the clinical effect of this agent at present, since the result of the clinical experiment with this agent has not been so far found in any literature. It may be, therefore, considered that the relationship between the effect in eggs, mice and human experiments is still ambiguous from the finidng with Caprochlorone.

Magrassi(7) published that Xenalamine hydrochloride was ineffective on PR-8 strain in eggs experiments, but effective in mice and clinical experiments. We found, however, that this agent did not show any significant effect on PR-8 strain in chorioallantoic membrane culture and mice experiments. Magrassi explained the mode of action of this agent as follows: this agent converts influenza virus in mouse lung to "virus mutilato" affording not any pathogenity and blood cell agglutination by acting on host cells, and this multilato contributes to the effect of this agent by producing antibodies in host cells and interfering with the viral infection. This speculation is appearently of interest as adding a new possibility to the virus chemotherapy, but we wonder whether we set due value on this hypothesis, since we could not find any significant effect of this agent in our repeated mice experiments. Accordingly, it may be said that the findings of Magrassi with Xenalamine hydrochloride also do not clarify the relationship between the effect of eggs, mice and human experiments.

Melander(6) claimed that $A B O B$ showed an effect on influenza virus in mice by lowering the consolidation of mice lungs and also an effect in embryonated eggs. We could not, however, find such the effect on PR-8 and Tokyo-57 strain in embryonated chorioallantoic membrane culture and mice experiments. Liu(11) reported that the inhibitory effect on the multiplication of the virus was observed with a dose more than $500 \mathrm{mg} / \mathrm{egg}$ of $\mathrm{ABOB}$.

Fukumi(12) described that the effect of $A B O B$ might be decided within the experimental error, because Melander conducted experiments using too small amount of the virus. Ishida(13) reported that $A B O B$ did not show any effect on the virus in eggs and mice experiments, but a greater amount of $A B O B$ exerted an inhibitory effect in parainfluenza-HeLa cell system.

All of the finding of Liu, Fukumi, Ishida and us suggest that ABOB is ineffective on influenza virus, in a concentration of chemotherapeutically significance, Recently, Jackson et al, ${ }^{(8)}$ reported that $A B O B$ was entirely ineffective in 
clinical trials with the viral examination. It may be, therefore, said that the finding with $\mathrm{ABOB}$ did not contribute to the correlation between the effect in eggs, mice and human experiments.

In contrast with the above findings, we obtained 4-amino-6-morpholino-striazine-2-carboxyguanidide as an antiinfluenzal agent by chorioallantoic membrane culture and mice experiments. The clinical test with this agent is of interest for finding an antiinfluenzal drug and acquiring the knowledge as for the correlation between the effects in eggs, mice and human experiments.

The clinical trials with this agent will be conducted in Japan, in the near future.

\section{REFERENCES}

1. Tamm, I.: Selective Inhibition of Virus Multiplication. in Cowan, S.T. and Rowatt, E. ed: Strategy of Chemotherapy. New York, Cambridge University Press, 1958. P. $178 \sim 211$.

2. Ueda, T., and Toyoshima, S.: Advances in Synthetic Antiviral Agent Research. Papers were read at the Symposium of Antiviral Agents in Tokyo, March, 1962.

3. Ledinko, N. and Perry, B.: Studies with Influenza Virus B of Recent Human Origin. I. Adaptation to the Mouse Lung. J. Immunol., 74: 371, 1955.

4. Ogasawara, K., and Nakayama, M.: Mechanism of Production of Pulmonary Consolidation in Mice by Newcastle Disease Virus (NDV). Virology, 6: 288, 1958.

5. Liu, O. C., Carter, J. E., Malsberger, R. G., DeSanctis, A. N. and Hampil, B.: Studies on the Chemotherapy of Viral Infections. II. The Effect of Caprochlorone on Influenza Virus Infection in Mice. J. Immunol., 78: 222, 1957.

6. Melander, B.: N1, N1-Anhydrobis ( $\varepsilon$-hydroxyethyl) biguanide- $\mathrm{HCl}$ (ABOB) in Prophylaxis and Suppression of Experimental Influenza. Antibiotic Chemotherapy, 10: 34, 1960.

7. Magrassi, F.: Possibility for the Production of the Synthetic Antiviral Agents. Virus (JAPAN), 11: 145, 1961.

8. Jackson, G. G., Muldon, R. L., Akers, L. W., Liu, O., Johnson, G. and Engel, C.: Effect of $\mathrm{N}^{1} \mathrm{~N}^{1}$-Anhydrobis-( $\beta$-hydroxyethyl) -biguanide Hydrochloride on Asian Influenza Virua in Voluntheers. in Finland, M. and Savage, G. M. ed: Antimicrobial Agents and Chemotherapy. Detroit, American Society for Microbiology 1961. P. 883 891.

9. Ueda, T. and Toyoshima, S.: Fundamental Chemotherapeutic Studies and Clinical Effect of PANS. Keio J. Med., 5: 123, 1956.

10. Yokota, M., Kobashi, K., Yokota, T., Takata, O., Nakanishi, R., Nakayama, D. and Watanabe, M.: Clinical Evolution of various Drugs on Japanese B Encephalitis. J. Japanese Assoc. Infec. Dis., 27: 235, 1953. (in Japanese)

11. Liu, O. and Engel, C. G.: Antiviral Effect of ABOB in Chick Embryo. Papers were read at the 28th International Symposium of Chemotherapy in Milano, 1960.

12. Fukumi, H.: On Antiviral Agents. Yakugyo Jiho, 4: 38, 1962. (in Japanese)

13. Ishida, N.: The Screening Methods for Antiviral Agent Research. Papers were read at the Symposium of Antiviral Agents in Tokyo, March, 1962. 